\title{
STEADY-STATE VS. PULSED EXCITATION RECOMBINATION KINETICS: SIMULATIONS AND EXPERIMENTS ON PORES, POWDERS AND RANDOM MEDIA
}

\author{
S.J. PARUS, Z-Y. SHI and R. KOPELMAN \\ Department of Chemistry, University of Michigan, Ann Arbor, Michigan 48109, USA
}

\begin{abstract}
The non-classical kinetics of exciton recombination in restricted geometries provides the foundation for a new experimental technique of probing the exciton dynamics and the sample topology. The phosphorescence and delayed fluorescence decays exhibit a dramatic dependence on the duration of the excitation. The comparison of pulsed and steady-state excitation provides information on the local topology of the sample and on the average hopping time of the exciton and the exciton diffusion length. This is possible because the distribution of the exciton population is non-Poissonian under steady-state excitation conditions. In addition, the pulse-created distribution also loses its Poissonian character with time. The experimental systems are: 1) isotopic mixed naphthalene crystals above and below percolation; 2) naphthalene crystalline powder; 3) naphthalene embedded into porous glass. Except for the mixed crystals above the percolation concentration, all samples exhibit non-classical effects. The interpretation is aided by Monte Carlo simulations.
\end{abstract}

\section{Introduction}

The compactness of random walks in low dimensions causes the formation of "depletion zones" around each particle [1]. These depletion zones give rise to anomalous distributions. To illustrate the experimental ramifications of the kinetic self-ordering of reaction particles we briefly present new experimental studies of exciton fusion $(\mathrm{A}+\mathrm{A} \rightarrow h \nu)$. This results in a new method for media characterization.

Figure 1 shows an example of an $\mathrm{A}+\mathrm{A} \rightarrow 0$ reaction in one dimension. The steady-state distribution is described by the approximate functional form:

$P(r)=B r \mathrm{e}^{-\beta(r-1)}, \quad B-\rho^{2}$,

where $\rho$ is the density, rather than by Hertz function [2]

$P(r)=A \mathrm{e}^{-\alpha(r-1)}, \quad A \sim \rho$,

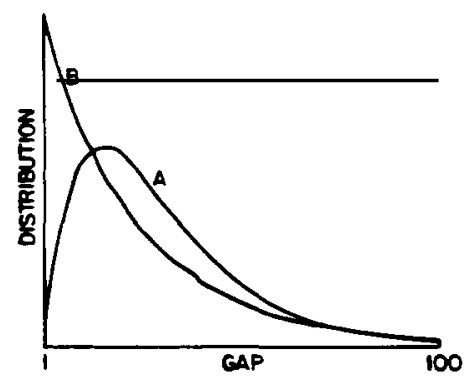

Fig. 1. Nearest neighbor distance ("Gap", in lattice units) distributions in one dimension: (A) A kinetically ordered particle ensemble (KOPE) for the steady-state reaction $\mathrm{A}+\mathrm{A} \rightarrow 0$. (B) A random particle ensemble (Hertz distribution). For simulation details, see ref. [3]. which is the form of the random (Poisson) distribution. Note that we consider hard-core particles and that we normalize the lattice-unit to unity.

\section{Monte Carlo simulations}

The experimental exciton reaction: Triplet + Triplet $\rightarrow$ Triplet or Singlet, where Singlet $\rightarrow$ Triplet or fluorescence, was simulated by reactive random walkers $(A+$ $\mathrm{A} \rightarrow 0$ and $\mathrm{A}+\mathrm{A} \rightarrow \mathrm{A}$ ) on a variety of lattices. Comparisons were made between different initial conditions. Steady state conditions were generated by adding several new walkers per step until the total number of walkers was constant. Pulsed conditions were then studied by adding, in one step, the same number of walkers that exist at steady state to produce an initial uniformly random distribution (fig. 1). In both cases, after stopping the addition of walkers $(t=0)$, the number of walkers or reactants remaining at time $t$ (monitored experimentally by phosphorescence) and the number of annihilations or reaction rate at time $t$ (monitored experimentally by delayed fluorescence) were monitored on the computer. While the initial $(t=0)$ global densities are equal for both forms of reactant generation, this does not guarantee equal reaction rates at $t>0$ or equal global densities at $t>0$. Examples are given in fig. 2 .

\section{Experiments: time modulation technique}

Triplet fusion experiments were performed on naphthalene impregnated porous glass (Vycor), naphthalene powder and perfectly crystalline isotopic alloys of naph- 


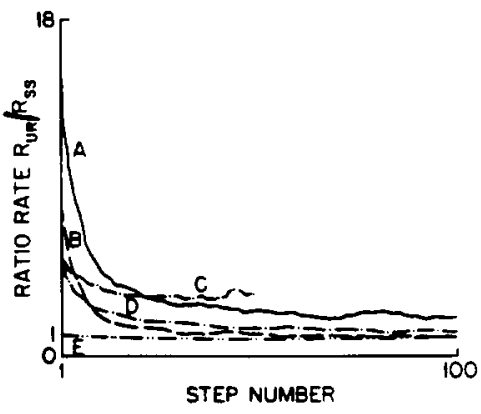

Fig. 2. Simulation examples of time-modulation technique. Ratio of annihilation reaction rates $R_{\mathrm{ur}} / R_{\mathrm{ss}}$ (uniformly random pulse over steady-state generated population) vs. step number, for the simulation $\mathrm{A}+\mathrm{A}=0$ on various lattices (total number of sites $=30000$ ). Curve $A$, one-dimensional islands (20 sites each); curve $B$, one-dimensional continuous chain; curve $C$, three-dimensional islands $(3 \times 3 \times 3$ sites each $)$; curve $\mathrm{D}$, three-dimensional percolation clusters (cubic; $40 \%$ occupation, all clusters); curve $\mathrm{E}$, three-dimensional cube.

thalene $\left(\mathrm{C}_{10} \mathrm{H}_{8}: \mathrm{C}_{10} \mathrm{D}_{8}\right)$. These samples cover a dimensionality range between one and three for exciton transport. The time dependence of the naphthalene triplettriplet exciton annihilation reaction rate was monitored, as usual, via the delayed fluorescence emission. Phosphorescence emission corresponds to the instantaneous triplet reactant concentration (global density). The dependence of the relative decay rates and intensities of the emissions on initial excitation duration was examined. "Time modulation" pertains to the modulation of this excitation duration. A random population of excitons was produced by pulsed excitation (e.g. $5 \mathrm{~ms}$ duration) from a mechanically shuttered xenon arc lamp.

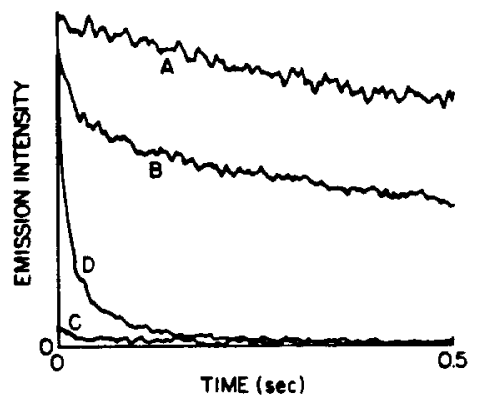

Fig. 3. Relative intensities of phosphorescence and of delayed fluorescence decays from naphthalene in porous Vycor glass at $18 \mathrm{~K}$ following steady-state $(11 \mathrm{~s}$ duration) or pulsed $(20 \mathrm{~ms}$ duration) excitation $(319 \mathrm{~nm}$ ). Curve A, phosphorescence from steady-state excitation with a number of 2.0 neutral density filter to equalize its initial intensity to that of curve B; curve B, phosphorescence from pulsed excitation; curve $C$, delayed fluorescence from conditions in curve $A$; curve $D$, delayed fluorescence from conditions in curve B. Note that the intensity scale for curves $C$ and $D$ is different from that for curves $A$ and $B$.

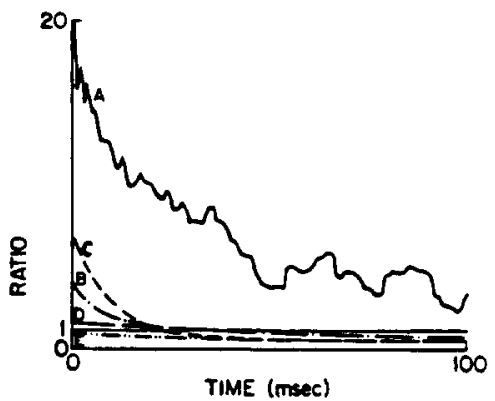

Fig. 4. Ratio of delayed fluorescences: pulsed vs. steady-state excitation, with equalized initial phosphorescence intensities. Curve A, naphthalene in porous Vycor glass; curve B, naphthalene powder; curve C, $5 \%$ isotopic mixed naphthalene crystal; curve D, $11 \%$ isotopic mixed crystal; curve E, 39\% isotopic mixed crystal. Note that the deviations from the classical value (unity) are up to $2000 \%$ and exceed by far the "noise".

The steady state population was created by leaving the shutter open for several seconds (which is longer than the time required to establish a constant phosphorescence signal). Neutral density filters werre used to give a phosphorescence intensity at time zero (the closing time of the shutter) equal to that obtained from the pulsed excitation. This ensures equal initial global exciton densities. However, this equalization is not sufficient to assure equal initial annihilation kinetics. Both the phosphorescence and the delayed fluorescence time decays are quite different for the pulsed and steady state cases. Fig. 3 shows the results for naphthalene impregnated porous glass. Similar results are obtained for naphthalene powder and for low concentration naphthalene single crystal isotopic alloys $\left(\mathrm{C}_{10} \mathrm{H}_{8}\right.$ below percolation [1]). These samples also exhibited low-dimensional exciton transport in other studies. On the other hand, no such differences in intensities or decay rates are observed for high concentration alloys (above percolation) or for nearly perfect naphthalene crystals. Above percolation, the single crystal alloy exhibits classical rather than geometrically-restricted transport characteristics [1] and no self-ordering is expected. This range of behaviors is shown in fig. 4 .

\section{Acknowledgement}

This work was supported by the National Science Foundation, Grant No. DMR-8801120.

\section{References}

[1] R. Kopelman, Science 241 (1988) 1623.

[2] P. Hertz, Math. Ann. 6 (1909) 387.

[3] S.J. Parus and R. Kopelman, Phys. Rev. B39 (1989) 889. 